\section{Tablettenimmuntherapie bewährt sich}

\author{
Allergentabletten sollen in naher Zukunft die spezifische Immun- \\ therapie weiter vereinfachen und damit auch einem größeren \\ Patientenkreis erschließen. Wirksamkeit und Sicherheit dieses Ver- \\ fahrens wurden in drei aktuell publizierten Studien untersucht.
}

E ine neu entwickelte Allergentablette - $\left(\right.$ GRAZAX $\left.^{\circledR}\right)$ zur sublingualen Anwendung löst sich bei Kontakt mit der Mundschleimhaut rasch auf und setzt eine definierte Grasallergendosis frei. Der geplanten Markteinführung dieser neuen Form der Hyposensibilisierung geht ein großes Studienprogramm voraus.

\section{Mehr Lebensqualität bei Rhinitis}

Eine internationale, multizentrische Studie untersuchte die Wirksamkeit der Tablettenimmuntherapie bei allergischer Rhinitis [1]. 855 Patienten zwischen 18 und 65 Jahren, die unter saisonaler Rhinokonjunktivitis litten, nahmen an der Studie teil. Bei allen war durch Pricktest und Bestimmung des spezifischen IgE eine Sensibilisierung gegen Wiesenlieschgras (Phleum pratense) nachgewiesen worden. Über im Mittel 18 Wochen erhielten die Teilnehmer randomisiert Tabletten in einer von drei Dosierungen des Graspollenallergens (2.500, 25.000 und 75.000 SQ-T) oder Plazebo zur täglichen Einnahme. Ausgewertet werden konnten die Daten von 790 Patienten.

Während der Pollensaison verringerten sich unter der höchsten Dosierung Symptom- und Medikationsscore moderat $(-16 \%$ bzw. $-28 \%)$ im Vergleich zu Plazebo ( $p=0,071$ bzw. $p=0,047)$. Signifikant verbesserte sich die auf die Rhinokonjunktivitis bezogene Lebensqualität ( $\mathrm{p}=0,006)$, und die Zahl der als ,gut“ bezeichneten Tage stieg signifikant an $(\mathrm{p}=0,041)$. Am deutlichsten waren die Effekte bei Patienten, die - wie empfohlen - bereits acht Wochen vor der Pollensaison ihre Behandlung begonnen hatten. Bei ihnen reduzierten sich die Symptome um 21\% ( $=0,002)$ und der Medikamentenverbrauch um 29\% ( $\mathrm{p}=0,012)$ siginifikant gegenüber Plazebo.

\section{Auch bei leichtem Asthma sicher}

Nicht selten treten allergische Rhinokonjunktivitis und Asthma bronchiale

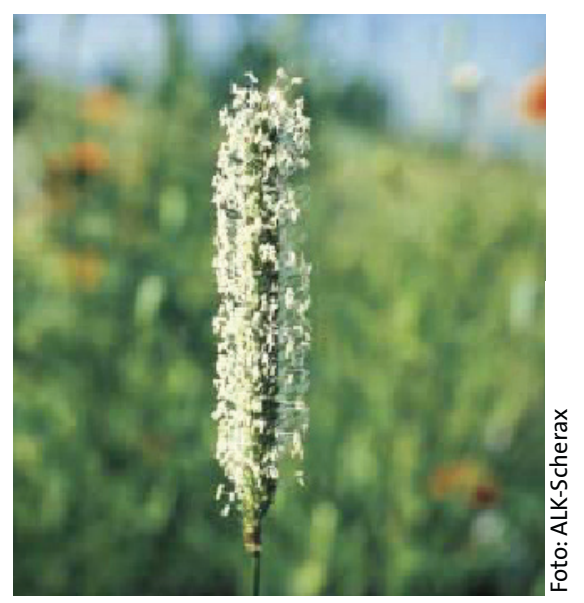

Die Tablettenimmuntherapie mit Allergenen des Wiesenlieschgrases hat ihre Wirksamkeit und Sicherheit bewiesen.

gemeinsam auf. Auch bei diesen Patienten sollte die Immuntherapie per Tablette Wirksamkeit und Sicherheit beweisen. Dazu wurden in einer Doppelblindstudie 114 auf Phleum pratense allergisch reagierende Patienten mit Rhinokonjunktivitis und Asthma bronchiale im Verhältnis zwei zu eins auf Verum und Plazebo randomisiert aufgeteilt [2]. Die Immuntherapie mit Tabletten (75.000 SQ-T, entspricht $15 \mu \mathrm{g} \mathrm{Phl} \mathrm{p} \mathrm{5)} \mathrm{erfolgte}$ 10 bis 14 Wochen vor Beginn der Pollensaison.

Es zeigte sich, dass der vor der Pollensaison niedrige Symptomscore und die relativ geringe Asthmamedikation während der Pollensaison in beiden Gruppen nur gering zunahmen, und zwar in Verum- und Plazebogruppe vergleichbar. Es kam zu keinen Asthmaexazerbationen durch das Verum. Die Teilnehmer der Immuntherapiegruppe litten aber signifikant weniger an Rhinokonjunktivitis-Symptomen $\quad(-37 \%$, $\mathrm{p}=0,004)$ und verbrauchten um $41 \%$ weniger Heuschnupfenmedikamente $(\mathrm{p}=0,036)$ als die Teilnehmer der Plazebogruppe. Der Anteil der während der Pollensaison als gut bezeichneten
Tage nahm nach der Tablettenimmuntherapie um 54\% gegenüber der Vergleichsgruppe zu.

\section{Tabletten im Sicherheitstest}

Die Sicherheit von sieben verschiedenen Dosierungen der Tablettenimmuntherapie mit Graspollenallergen (25.000 bis 1.000.000 SQ-T) untersuchte eine Phase-I-Studie an insgesamt 84 Studienteilnehmern [3]. Bei keiner Dosierung traten schwere, systemische oder zum Studienabbruch führende unerwünschte Wirkungen auf. Die Inzidenz von milden oder moderaten lokalen Nebenwirkungen lag bei $74 \%$ und war dosisabhängig. Die häufigsten unerwünschten Wirkungen waren Reizungen des Rachens und Juckreiz in Mund und Ohren. Meist begannen diese Missempfindungen kurz nach der Einnahme und dauerten nur Minuten oder wenige Stunden an. Auch in den beiden zuerst genannten Wirksamkeitsstudien traten keine schwerwiegenden Sicherheitsprobleme auf.

Fazit: Die Studien belegen einen dosisabhängigen Effekt der Tablettenimmuntherapie mit Graspollenallergen und eine signifikante Wirksamkeit der Dosierung 75.000 SQ-T bei Rhinokonjunktivitis. Dabei konnten nicht nur eine Verringerung von Symptom- und Medikationsscore, sondern auch ein positiver Effekt auf die Lebensqualität gezeigt werden. Aufgrund der milden und transienten lokalen Nebenwirkungen könnte die spezifische Immuntherapie mit Tabletten eine neue Möglichkeit zur Hyposensibilisierung bei Rhinokonjunktivitis durch Graspollenallergie sein.

1. Durham SR et al. Sublingual immunotherapy with once-daily grass allergen tablets: A randomized controlled trial in seasonal allergic rhinoconjunctivitis.J Allergy Clin Immunology 2006; 117: 802-9

2. Dahl R et al. Specific immunotherapy with SO standardized grass allergen tablets in asthmatics with rhinoconjunctivitis. Allergy 2006; 61: 185-90

3. Kleine-Tebbe J et al. Safety of a SOstandardised grass allergen tablet for sublingual immunotherapy: a randomized, plazebo-controlled trial. Allergy 2006; 61: 181-4 